\title{
Volviendo al tema de la democracia
}

\author{
Programa de las Naciones Unidas para el Desarrollo (pnud),
} DaNTE CAPUTO (DIRECTOR DEL PROYECTO) (2004), LA DEMOCRACIA EN AmÉRICA LATINA. HACIA UNA DEMOCRACIA DE CIUDADANAS Y CIUDADANOS, pnud, Aguilar, Altea, Taurus, Alfaguara, Buenos Aires, 255 pp., ISBN: 987-04-0032-9.

En poco más de dos décadas, los países de América Latina fueron adaptando sus regímenes políticos a los preceptos democráticos. No fue este, sin duda, un hecho menor. En la segunda mitad del siglo xx habían arreciado sobre la región cruentas dictaduras que, además de haber contado muertos de a miles, dejaron a la mayoría de los países sumidos en una profunda crisis económica, con una deuda externa impagable, y social. La recuperación, en unos casos, y la instauración de la democracia, en otros, resultó imprescindible para la viabilidad de estas sociedades.

El recibimiento que los latinoamericanos le fueron dando a sus respectivas transiciones no pudo ser más venturoso. El oprobio vivido en las décadas previas había alcanzado su propia cotidianidad, por lo que no se remitía exclusivamente al sistema político. Esto le dio al régimen un bono de legitimidad bastante amplio. No obstante ello, la tarea no era sencilla. Las nuevas democracias debieron enfrentar una doble tensión. Por un lado, las democracias recién instaladas tuvieron que afrontar la tarea de la democratización. Establecer nuevas reglas del juego, modernizar instituciones y procedimientos, dinamizar la vida política, difundir valores democráticos, reinstitucionalizar a los partidos políticos, etc. Por el otro, estaban obligadas a encarar el inmediato problema económico y social. A pesar del bono de legitimidad, las nuevas democracias no podían descansar sólo en ello; requerían, y pronto se notó, eficiencia en la gestión.

La tensión a la que fue sometida la democracia en América Latina, además de las visiones reduccionistas dominantes, hicieron de ella un régimen recluido en lo electoral. El retorno de las democracias a la región se produjo poco después de la crisis de los Estados de bienestar en los países del Primer Mundo. Para entonces el diagnóstico dominante había sido formulado mediante el Informe de la Comisión Trilateral de 1975 denominado La gobernabilidad de la democracia. 
El informe, encargado por dicha comisión a tres destacados politólogos, Samuel Huntington, Michel Crozier y Joji Watanuki, establecía que la crisis que azotaba a los países del Primer Mundo se debía a la sobrecarga de demandas por parte de los ciudadanos hacia el Estado. La concentración en la agenda de actividades que 'debía' desarrollar la sociedad hizo que todas las demandas ciudadanas se dirigieran al Estado, lo que lo orilló a una situación de ingobernabilidad política y anomia social. La solución recomendada se orientaba a la disminución de las expectativas ciudadanas y a la reducción de los servicios sociales del gobierno.

Semejante diagnóstico para las consolidadas democracias centrales no podía más que aterrorizar a las débiles e incipientes democracias latinoamericanas que debían afrontar rezagos sociales mucho más apremiantes. Las recomendaciones de menos democracia, menos política y menos Estado fueron difundidas por los políticos e intelectuales locales de manera inmediata con el objeto de garantizar la gobernabilidad. Los líderes locales se horrorizaron por el peligro de un desbordamiento social que condujera a un retorno del autoritarismo. Así, sociedades acorraladas por sus propias condiciones de subsistencia, que habían luchado por la democracia como una forma de recuperar su propia dignidad, pronto se vieron acorraladas por argumentos que, aunque renovados, sabían a poco democráticos.

El discurso de la gobernabilidad se apoderó también de los organismos multilaterales de crédito, lo cual impactó aún de manera más directa sobre nuestras noveles democracias. El Informe sobre el Desarrollo Humano que el Banco Mundial dio a conocer en 1989, y su impacto por medio de las recomendaciones para la renegociación de la deuda externa y los proyectos de asistencia técnica a los gobiernos de la región, ubicaron el problema del desarrollo en el excesivo tamaño del Estado y en el abultado déficit fiscal que hacían imposible asumir el pago de los compromisos de la deuda y, con ello, el acceso a las indispensables fuentes de financiamiento.

$\mathrm{Al}$ año siguiente, el foro que derivó en el documento denominado Consenso de Washington profundizó esta visión dominante. El foro, al que acudieron académicos y funcionarios públicos de América Latina y de los organismos internacionales, concluyó con diez recomendaciones para superar la crisis de la región, cinco de ellas destinadas a la estabilización económica a través 
de políticas monetarias ortodoxas, y las otras cinco a la reducción sustancial de la dimensión y el papel del Estado.

Esta visión impulsada por los organismos internacionales eliminó del discurso incluso las definiciones en términos de la gobernabilidad, planteadas por el informe de la Comisión Trilateral, que ponía en el centro de la cuestión la crisis fiscal y financiera. Desde principios de la década de los noventa, los problemas vinculados a la definición del Estado y la relación de la economía con el Estado y la sociedad abandonaron la agenda de la democracia en la región para enfocarse exclusivamente en la cuestión monetaria y fiscal.

De tal forma que si para inicios de la transición el dilema giraba en torno a los peligros derivados de los excesos de democracia, para 1990 se centraba exclusivamente en la eficiencia económica del Estado.

La profundidad de la crisis y la contundencia de las recomendaciones obligaron a enfocar todos los esfuerzos gubernamentales y sociales a la política económica. Los encargados de esas carteras, a diferencia de lo ocurrido en épocas previas, se volvieron los personajes clave de sus respectivos gabinetes.

Si bien posteriormente comenzaron a flexibilizarse las recomendaciones de política económica que permitieron una reinterpretación acerca del papel del Estado, lo cierto es que los debates en relación con el papel de la política y la extensión de la democracia no corrieron la misma suerte. ${ }^{1}$ El Estado ideal dejó de ser el mínimo, pero la reconsideración en torno a su dimensión y sus funciones no se planteó en la arena política y ni en la necesidad de acuerdos.

El informe que reseño en esta ocasión es el primer esfuerzo procedente de un organismo internacional que pretende retomar este debate inacabado. Consiste, fundamentalmente, en una reinterpretación de la crisis latinoamericana, tal vez una década más tarde de lo aconsejado, y una recolocación que lleva a plantear el papel de la política y de la democracia en la crisis (que ya deja de considerarse como exclusivamente económica) y en su superación.

Se trata de un documento de poco más de 200 páginas, dividido en tres secciones, además de prólogos, resúmenes y apéndices, en el que se plantean tres preguntas fundamentales: ¿Cuál

${ }^{1}$ El Informe sobre el Desarrollo Humano que el Banco Mundial presentó en 1993 y el informe denominado El Estado en un mundo en transformación que el mencionado organismo publicó en 1997 son prueba de ello. 
es el estado de la democracia en América Latina? ¿Cuáles son las percepciones y cuán fuerte es el apoyo de líderes y ciudadanos a la democracia? ¿Cuáles serían los principales temas para un debate orientado a lograr un mayor avance en la democracia de ciudadanos?

Para responder a la primera pregunta, el informe parte de una definición de democracia como forma de organización social que tiene su fundamento normativo en la definición del ser humano como sujeto portador de derechos y como ciudadano, fuente de autoridad, sobre quien descansa la soberanía popular que se expresa en elecciones libres e institucionalizadas. Se trata de un sujeto responsable y autónomo, de un individuo capaz de elegir, quien, en tanto ciudadano y fuente de la que emana toda autoridad, no puede ser tratado como súbdito.

Hasta aquí la definición no se aparta de la que postulaba el liberalismo decimonónico, y resulta muy acorde con los planteamientos de los defensores del Estado mínimo, si no fuera que considera al sujeto no sólo como portador de derechos políticos, sino también como portador de derechos que hacen posible tales derechos políticos; y a la democracia como un régimen político que requiere, para ser tal, derechos políticos pero también derechos civiles (fundamentalmente la libertad para elegir y la equidad) sin los cuales la ciudadanía es superficial, y sociales, definidos como la base de condiciones para eliminar las privaciones que impiden el ejercicio de las opciones responsables y las libertades que implican.

Desde esta perspectiva, la democracia no tiene sólo una dimensión institucional. Las reglas que implica el régimen político democrático son sólo condiciones necesarias pero no suficientes para hablar de democracia. La democracia va más allá del régimen político

A partir de su definición, el informe intenta describir la situación actual de la democracia en América Latina mediante un conjunto de indicadores que abarcan cada una de las dimensiones de la ciudadanía integral.

Los indicadores de desarrollo de la ciudadanía política se encuentran descritos en el cuadro 1. El balance que arroja la región en relación con estos indicadores es, de acuerdo con el informe, bastante positivo en la medida que: 1) es ampliamente reconocido el derecho al voto; 2) la práctica de las elecciones limpias constituye un patrón generalizado; 3) la imposición de las elecciones como medio de acceso a cargos públicos registra un nota- 
ble avance; 4) la participación electoral es moderadamente amplia; 5) no existen marcadas barreras de acceso para la competencia política; 6) se percibe una tendencia positiva a la introducción de normas para una mayor inclusión, y 7) existe una considerable proporcionalidad en los sistemas electorales. Los aspectos marcados como pendientes de atención son: a) el corto alcance de la legislación sobre el financiamiento de los partidos; b) la persistente supremacía del Poder Ejecutivo sobre el Legislativo; c) las interferencias sobre el Poder Judicial; $d$ ) el desarrollo desigual de los mecanismos de democracia directa, y e) la poca independencia de las agencias especializadas en el control de la gestión pública.

\section{Cuadro 1}

\begin{tabular}{lll}
\hline Índice de democracia & $\begin{array}{l}\text { Elecciones libres } \\
\text { electoral } \\
\text { Elecciones limpias } \\
\text { Cargos públicos electos }\end{array}$ \\
Ciudadanía política & Control de gestión & $\begin{array}{l}\text { División de poderes } \\
\text { Democracia directa } \\
\text { Agencias especializadas }\end{array}$ \\
& $\begin{array}{l}\text { Clecciones internas en los } \\
\text { partidos }\end{array}$ \\
selección de candidatos & $\begin{array}{l}\text { Barreras de entrada a los } \\
\text { partidos políticos } \\
\text { Monopolio partidista de las } \\
\text { candidaturas }\end{array}$ \\
\hline
\end{tabular}

El cuadro 2 resume los indicadores que utiliza el informe para la evaluación del desarrollo de la ciudadanía civil. Los resultados de la valoración de esta dimensión de la ciudadanía integral son menos alentadores que en los de la dimensión anterior. Los datos aportados por el informe apuntan a: 1) ciertos logros significativos en el reconocimiento legal de los derechos civiles en general, de las mujeres y de los pueblos indígenas; 2) avances en el respeto a los derechos humanos y la libertad de prensa; 3) disparidades en la aplicación de la igualdad legal y la protección contra la discriminación; 4) altos niveles de inseguridad ciudadana que limitan el derecho a la vida, a la integridad física y a la seguridad, y 5) violaciones de los derechos de los acusados y los presos que no evita el funcionamiento de la administración de justicia. 


\section{Cuadro 2}

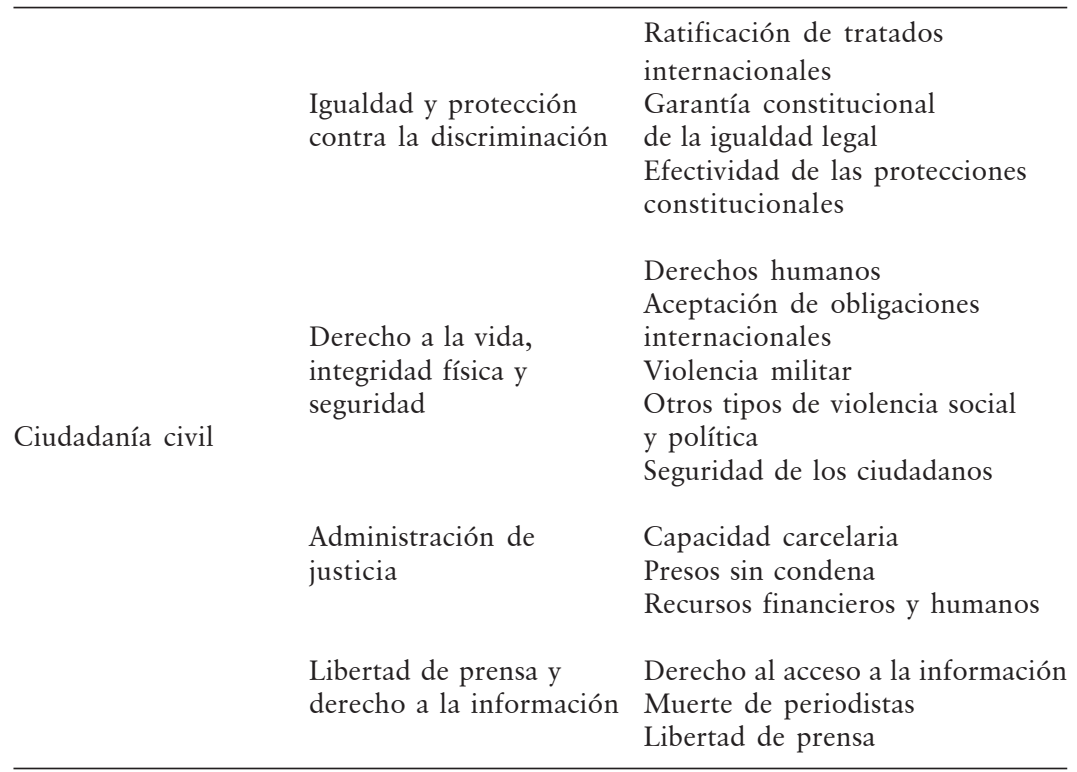

Por lo que se refiere a los derechos sociales, los datos aportados para los indicadores utilizados por el informe, resumidos en el cuadro 3, muestran que esta es la dimensión de la ciudadanía integral que peores condiciones registra, por lo que se considera en el documento que es uno de los desafíos más urgentes que enfrenta la región.

\section{Cuadro 3}

\begin{tabular}{lll}
\hline \multirow{2}{*}{ Ciudadanía social } & Integración social & $\begin{array}{l}\text { Pobreza } \\
\text { Desigualdad } \\
\text { Empleo }\end{array}$ \\
& Necesidades básicas & $\begin{array}{l}\text { Educación } \\
\text { Salud }\end{array}$ \\
\hline
\end{tabular}

Para responder la segunda pregunta que se plantea el informe, se realizó una encuesta durante el mes de mayo de 2002 sobre las percepciones ciudadanas acerca de la democracia, así como una ronda de consultas a 213 líderes latinoamericanos, incluidos 41 presidentes y vicepresidentes.

Los resultados obtenidos de la aplicación del primer instrumento son los siguientes: 1) 43\% de los encuestados tenían una 
orientación prodemocrática, es así la orientación más extendida; 2) la alternativa entre desarrollo económico y democracia evidencia una tensión, y parecería que muchos prefieren la primera; 3) los entrevistados pertenecientes a países donde hay menores niveles de desigualdad social tienden a ser menos favorables a la democracia; 4) los ciudadanos con orientaciones no democráticas se encuentran mayoritariamente entre los sectores que tienen menor educación, una socialización proveniente de periodos autoritarios, una percepción de baja movilidad social respecto de sus padres y bajas expectativas en cuanto a una futura mejoría para sus hijos, y aquellos que tienen mayor desconfianza en las instituciones; 5) la mayoría de los ciudadanos no está desconectada de la vida política y social de sus países, y 6) en promedio, los demócratas tienden levemente a participar más activamente en la vida política de sus países.

Por su parte, la ronda de entrevistas a los líderes muestra las siguientes opiniones preponderantes: 1) América Latina ha dado pasos muy importantes en el camino hacia la democratización, el aumento de la participación y de los controles institucionales es reconocido como un paso decisivo; 2) toda la región es, al menos formalmente, democrática, la dimensión institucional no se ve como un epifenómeno de lo que realmente importa, sino como parte constitutiva de la democracia; 3) algunas amenazas tradicionales a las democracias latinoamericanas han desaparecido o se han debilitado significativamente; 4) han aparecido otras amenazas que siguen poniendo en cuestión la continuidad y expansión de la democracia, la más ostensible es el narcotráfico; 5) otras amenazas sobre la democracia latinoamericana son políticas, las más importantes son: la reducida autonomía de decisión de los poderes institucionales y el debilitamiento de los partidos políticos; 6) la crisis de los partidos no ocurre debido a una pérdida de la voluntad ciudadana de participación, antes bien, enfrentan un problema nuevo que combina tres elementos: una voluntad de mayor participación y control de poder político, un rechazo generalizado de los partidos como canales de participación y un traslado de la participación y el ejercicio de controles hacia otros tipos de organizaciones en general pertenecientes a la sociedad civil; 7) es importante tener partidos fuertes y gobiernos con capacidad de decisión; 8) un primer desafío de la democracia latinoamericana es encontrar soluciones políticas a sus problemas políticos; 9) un segundo desafío es encontrar soluciones a la desigualdad, la pobreza y la actual imposibilidad de 
acceso de gran parte de la población a los niveles de bienestar necesarios para el pleno ejercicio de los derechos.

Aportados los datos que arroja el estudio empírico, el informe se aboca a la definición de cuatro esferas que, en su evaluación, resultan indispensables para el desarrollo de la democracia en América Latina: política, Estado, economía y globalización.

El informe establece que existe un déficit de poder democrático en las democracias de la región. Define este poder como la capacidad de actuar de modo efectivo frente a los problemas para expandir la ciudadanía. Sostiene, asimismo, que para construirlo es indispensable la política: "que la política sea relevante, que proponga caminos para abordar los temas claves de la sociedad, que los emprenda con la firmeza de la voluntad de los líderes y ciudadanos y la sostenga con la idoneidad de los instrumentos para la acción colectiva, entre los cuales los partidos políticos son actores centrales pero no los únicos” (p. 181).

El informe propone que las democracias latinoamericanas tienen también un déficit de estatalidad. Las propuestas de los partidos y de los actores de la sociedad civil requieren la eficacia ejecutoria de las instituciones representativas y del gobierno del Estado. Pues "el poder democrático se construyen también desde la estatalidad. Detrás de todo derecho hay un Estado que lo garantiza. Y detrás de todo derecho trunco hay un Estado que no llega a tornarlo efectivo. Esta defección del Estado tiene que ver con la calidad de sus instituciones y, fundamentalmente, con el poder que fluye a través de ellas y la consecuente capacidad -O incapacidad- del Estado para llevar a cabo sus fines” (p. 181).

Los déficit de política y de estatalidad que postula el informe se combinan con los requerimientos de crecimiento económico para superar los rezagos documentados por el informe en materia de ciudadanía social y con otros desajustes igualmente analizados. El crecimiento económico no es un dato sin más, sus resultados, frecuentemente generadores de desigualdades, conjugado con los límites del Estado, "con la impotencia de la política para encarar las aspiraciones de la ciudadanía en poder democrático, con las tensiones de sociedades fracturadas, con la existencia de poderes fácticos que evaden la legalidad, trafican influencias y permean las más altas instancias de decisión, con la evidencia de una globalización que acota el espacio propio de la democracia al escamotear del campo de la voluntad ciudadana los temas centrales que atañen al futuro de la sociedad" (p. 182). 
En América Latina, donde los principales desequilibrios se manifiestan en la dimensión social de la ciudadanía, "la sustentabilidad del sistema y la resolución de la crisis de representación política dependen de nuestra capacidad para incorporar a la economía y sus opciones como un tema de la democracia [...] La economía es una cuestión de la democracia porque de ella depende el desarrollo de la ciudadanía social y porque genera y altera las relaciones de poder” (p. 191). Y viceversa:

existen cinco funciones que las instituciones públicas deberían ofrecer para que los mercados funcionen adecuadamente: la protección de los derechos de propiedad, la regulación del mercado, la estabilización macroeconómica, el seguro social y el manejo de los conflictos de interés [...] Los mercados requieren gobernabilidad y reglas. La buena gobernabilidad sólo se asegura por la vía de la democracia [...] por ello la agenda de la sustentabilidad democrática debe incluir, a riesgo de vaciarse de contenido, estas cuestiones de la economía, sus opciones y su diversidad (p. 192).

El último tema de la agenda planteado por el informe es el de la globalización. Al respecto señala que el debate se ha centrado en los asuntos financieros y comerciales, y margina el hecho de que con la globalización los poderes exteriores han dejado de ser exteriores; que condicionan las decisiones del Estado más allá de los ámbitos financiero y comercial; que abarcan las cuestiones políticas, de seguridad, de seguridad social, de educación y de salud. Por esta razón, prosigue, es necesario observar, por un lado, el impacto real en términos de la soberanía interior de los Estados y, por el otro, concebir las estrategias posibles para aumentar las capacidades nacionales y regionales, para que el poder nacional no se extinga en nombre de un incontrolable poder global.

En suma, el informe llama a fortalecer la democracia mediante: 1) la revaloración de la política; 2) la recuperación del papel constructivo de la política como ordenadora de las decisiones de la sociedad; 3) la expansión de la ciudadanía; 4) el robustecimiento de la participación ciudadana; 5) sistemas de partidos que se fortalezcan a partir de la eficacia, la transparencia y la responsabilidad; 6) el fortalecimiento de la sociedad civil; 7) un Estado capaz de conducir el rumbo general de la sociedad; 8) una economía congruente con la democracia, y 9) la construcción de espacios autónomos. 
Es, sin duda, loable el esfuerzo de recuperar el debate sobre la democracia en la región. Mucho más cuando se hace desde un organismo internacional, lo cual implica una posición como interlocutor mucho más influyente que la que se tiene desde la academia. Independientemente de los responsables de llevar a cabo este informe, lo cierto es que, desde el punto de vista de la institución que lo produce, es un esfuerzo tardío. El daño hecho en la década de los noventa a los países de la región es irreparable. No sólo sus consecuencias más connotadas son de por sí lamentables, su perjuicio fue igualmente grave sobre las mediaciones que hacen posible la vida democrática. Por supuesto que, como dice el refrán, más vale tarde que nunca; pero no basta con cambiar de perspectiva, resulta necesario asumir las responsabilidades de lo realizado anteriormente. No será posible el desarrollo de la ciudadanía integral sin condiciones especiales para su recuperación. Se requiere un esfuerzo y un compromiso de parte de este tipo de instituciones que todavía no se ha percibido con claridad, ni el informe aborda con nitidez y compromiso.

Como trabajo académico, el informe tiene, insisto, la virtud de reubicar el debate. No se trata de un trabajo totalmente original en este esfuerzo. La academia viene advirtiendo sobre los problemas de la consolidación democrática en la región desde hace ya tiempo, aunque, como se dijo, con un impacto muy limitado. Incluso desde el punto de vista del análisis mismo, se requiere documentar más eficientemente muchas de las afirmaciones vertidas. Vale, pues, tanto por lo que dice como por quien lo dice. Y esto no es poco.

Javier Arzuaga Magnoni Facultad de Ciencias Políticas y Administración Pública, UAEMéx

Correo-e: jaam@uaemex.mx

Javier Arzuaga Magnoni es doctor en ciencias sociales por la Universidad Autónoma Metropolitana-Xochimilco, y miembro del Sistema Nacional de Investigadores. Desde 1993 se desempeña como investigador del Centro de Estudios Avanzados de la Facultad de Ciencias Políticas y Administración Pública de la Universidad Autónoma del Estado de México. Sus líneas de investigación son competitividad y geografía electorales así como sistemas de partidos políticos en México. Es autor del libro Racionalidad empresarial. Los megaempresarios mexicanos, coeditado 
por Gernika y la Universidad Autónoma del Estado de México en 2004; en 2002 publicó en coautoría con Carlos Alberto Sara Gutiérrez "La alianza virtual. Razones del cambio en las elecciones presidenciales en el Estado de México", Economía, Sociedad y Territorio, El Colegio Mexiquense, A.C., vol. III, núm. 11, Zinacantepec, pp. 441-470; y en 2000 publicó en coautoría con Cecilia Gayet "El avance de la pluralidad electoral en los sistemas políticos locales del Estado de México", Apuntes Electorales, revista del Instituto Electoral del Estado de México, año 1, núm. 2, Toluca, pp. 191-200; asimismo, "Democracia y consenso: una apuesta al gobierno de lo local”, Espacios Públicos, año 4, núm. 7, febrero de 2001, Toluca, México; "Argumentos a favor de una democracia con adjetivos", Apuntes Electorales, año 1, núm. 3, diciembre de 2000, Toluca; y “1996: Elecciones municipales y legislativas”, en coautoría con Ernesto Emmerich en Ernesto Emmerich (coord.), El voto ciudadano en el Estado de México (1990-1997), Universidad Autónoma del Estado de México, Toluca. 